\title{
Calycosin induces apoptosis via p38-MAPK pathway-mediated activation of the mitochondrial apoptotic pathway in human osteosarcoma 143B cells
}

\author{
WEI TIAN, ZHI-WEI WANG, BAO-MING YUAN and YONG-GE BAO \\ Department of Orthopedics, Affiliated Hospital of Inner Mongolia University for The Nationalities, \\ Tongliao, Inner Mongolia 028007, P.R. China
}

Received January 12, 2020; Accepted July 28, 2020

DOI: $10.3892 / \mathrm{mmr} .2020 .11471$

\begin{abstract}
Previous studies have demonstrated that calycosin is a natural phytoestrogen with a similar structure to estrogen, which can inhibit cell proliferation and induce apoptosis in a variety of tumors. Calycosin exerts potential pharmacological effects on osteosarcoma cells by inducing apoptosis. The aim of the present study was to elucidate the specific molecular mechanism of calycosin-induced apoptosis in osteosarcoma cells. Cell proliferation was determined by an MTT assay. Annexin V/PI and JC-1 staining were used to detect apoptosis and mitochondrial dysfunction, respectively, by flow cytometry. Western blot analysis was used to detect the expression of caspases or mitochondrial proteins. The results revealed that calycosin reduced the cell viability of human osteosarcoma 143B cells, induced apoptosis and increased the loss of mitochondrial membrane potential (MMP). In addition, calycosin increased the expression of the proapoptotic antiapoptotic proteins cleaved caspase-3, cleaved caspase-9, cleaved poly(ADP-ribose) polymerase and $\mathrm{Bcl}-2$-associated $\mathrm{X}$ protein (Bax), and decreased the expression of the antiapoptotic proapoptotic protein $\mathrm{B}$-cell lymphoma-2 (Bcl-2), thus altering the $\mathrm{Bax} / \mathrm{Bcl}-2$ ratio. In addition, the expression levels of cytochrome $c$ were markedly decreased in the mitochondria and increased in the cytoplasm following calycosin treatment. Furthermore, calycosin treatment induced p38-mitogen-activated protein kinase (MAPK)
\end{abstract}

Correspondence to: Professor Yong-Ge Bao, Department of Orthopedics, Affiliated Hospital of Inner Mongolia University for The Nationalities, 1742 Holin He Street, Tongliao, Inner Mongolia 028007, P.R. China

E-mail: baoyongge@163.com

Abbreviations: MMP, mitochondrial membrane potential; mTOR, mammalian target of rapamycin; PI3K, phosphatidylinositol 3-kinase; MAPK, mitogen-activated protein kinase

Key words: calycosin, osteosarcoma, p38MAPK, mitochondrion, apoptosis phosphorylation, whereas the p38-MAPK inhibitor BIRB 796 markedly reversed cell viability, apoptosis and loss of MMP in 143B cells. These results suggested that calycosin inhibited osteosarcoma 143B cell growth via p38-MAPK regulation of mitochondrial-dependent intrinsic apoptotic pathways.

\section{Introduction}

Osteosarcoma is one of the most common malignant tumors, with a high incidence and metastasis rate, primarily occurring in children and adolescents (1). In Portugal, the rate for African Americans is 5.1-0.3\% per 1 million children aged 0-14 years (2). For several decades, the treatment of osteosarcoma has primarily included surgery, radiotherapy and chemotherapy, but the results of these treatment are not satisfactory (3). The challenge for anticancer research is to identify a novel alternative approach that effectively targets osteosarcoma cells by inducing apoptosis, combined with low toxicity (4).

Previous evidence has revealed that calycosin, a bioactive phytoestrogen isoflavone that is extracted from Trifolium pratense (red clover), possesses anti-allergic (5), anti-inflammatory (6), antifibrotic (7), anti-myocardial injury (8) and antitumor (9-11) properties. Calycosin may exert a potential antimetastatic effect on several types of cancer, including breast cancer, colorectal cancer, ovarian cancer and nasopharyngeal carcinoma $(9,10)$. It also promotes apoptosis and autophagy in adenocarcinoma cells via sirtuin 1 (SIRT1)/AMP-activated protein kinase (AMPK)-induced inhibition of the Akt/mammalian target of rapamycin (mTOR) pathway (11). It inhibits the phosphatidylinositol 3-kinase (PI3K)/Akt/mTOR pathway, which in turn leads to apoptosis of osteosarcoma cells (12). Mitogen-activated protein kinases (MAPKs) are one of the most important regulators of apoptotic cell death (13) and the MAPK pathway has been reported to be involved in the proliferation and apoptosis of osteosarcoma cells (14). Calycosin-induced apoptosis occurs in breast cancer cells through regulation of the p38-MAPK and PI3K/Akt pathways (15). Therefore, a potential functional mechanism through which the anti-osteosarcoma effects may be mediated is inhibition of the MAPK pathway leading to cell death. The aim of the present study was to investigate whether calycosin 
exerted antitumor effects on osteosarcoma cells by inducing apoptosis via the MAPK signaling pathway.

\section{Materials and methods}

Calycosin. Calycosin (purity 98\%; Tianjin JAHE Science and Technology Co., Ltd.) was diluted into a $250 \mu \mathrm{g} / \mathrm{ml}$ stock solution with DMSO (Sigma-Aldrich; Merck KGaA). BIRB 796 was purchased from R\&D Systems, Inc.

Cell culture. The human osteosarcoma cell line 143B and the human osteoblast cell line hFOB1.19 (Shanghai Institute of Biochemistry and Cell Biology) were incubated in DMEM (Gibco; Thermo Fisher Scientific, Inc.) supplemented with 10\% FBS (Gibco; Thermo Fisher Scientific, Inc.), and $100 \mathrm{U} / \mathrm{ml}$ penicillin and $100 \mu \mathrm{g} / \mathrm{ml}$ streptomycin in a humidified incubator containing $5 \% \mathrm{CO}_{2}$ at $37^{\circ} \mathrm{C}$. The medium was changed every $48 \mathrm{~h}$.

Cells were cultured separately for $24 \mathrm{~h}$, and were then treated with various concentrations of calycosin $(0-160 \mu \mathrm{g} / \mathrm{ml})$ at $37^{\circ} \mathrm{C}$ for $48 \mathrm{~h}$. In addition, the p38 inhibitor BIRB 796 (Beyotime Institute of Biotechnology) was applied to further explore the expression of key proteins in osteosarcoma 143B cells following treatment with calycosin. Specifically, the cells were incubated with $1 \mu \mathrm{M}$ BIRB 796 for $2 \mathrm{~h}$ at $37^{\circ} \mathrm{C}$, and then treated with calycosin $(20 \mu \mathrm{g} / \mathrm{ml})$ for $48 \mathrm{~h}$.

MTT assay. Cell viability was determined by an MTT assay (Sigma-Aldrich; Merck KGaA). Cells were harvested using $0.25 \%$ trypsin and seeded into 96 -well plates at a density of $3 \times 10^{4}$ cells/well at $37^{\circ} \mathrm{C}$ for $24 \mathrm{~h}$. Then, the purple formazan was dissolved with $100 \mu \mathrm{l}$ DMSO, and cells were allowed to shake for $10 \mathrm{~min}$ on a mini shaker, and analyzed in a multi-well-plate reader at $570 \mathrm{~nm}$ on a microplate reader (Thermo Fisher Scientific, Inc.). The proliferation rate (\%) was calculated as follows: Optical density (OD) treatment group/OD control $\times 100 \%$.

Annexin V/PI staining assay. Early + late cell apoptosis was detected using flow cytometry. Cells were collected and washed twice with PBS. FITC-conjugated Annexin V (FITC-V) and PI double staining was used to identify cells (BD Biosciences) according to the manufacturer's instructions. Data were obtained and analyzed using a FACSCanto ${ }^{\mathrm{TM}}$ flow cytometer (Beckman Coulter, Inc.) with CellQuest software (version 5.1; BD Biosciences).

JC-1 staining assay. Mitochondrial membrane potential (MMP) monitoring was performed using the JC-1 MMP detection kit (Beyotime Institute of Biotechnology), following the manufacturer's protocol. Cells were collected, incubated with $\mathrm{JC}-1$ at $37^{\circ} \mathrm{C}$ for $20 \mathrm{~min}$, and then washed twice with PBS. JC-1 green and red fluorescence were recorded by flow using a FACSCanto ${ }^{\mathrm{TM}}$ flow cytometer (Beckman Coulter, Inc.) as aforementioned at 527 and $590 \mathrm{~nm}$, respectively. Mitochondrial depolarization was manifested by a decrease in $\mathrm{red} / \mathrm{green}$ fluorescence intensity ratio.

Western blot analysis. Protein expression was detected by western blotting. Cells were harvested, following which proteins were extracted from the cells using a cocktail of cell lysis buffer supplemented with protease inhibitor (Beyotime Institute of Biotechnology) in the ratio of 100-200 $\mu$ l of lysate to each well of the 6-well plate. The lysates were centrifuged at $4^{\circ} \mathrm{C}$ at $700 \mathrm{x} \mathrm{g}$ for $10 \mathrm{~min}$ and collected. Then, the total protein was measured with a bicinchoninic protein assay kit (Tiangen Biotech Co., Ltd.). The samples $(20 \mu \mathrm{g})$ were separated via SDS-PAGE on a $12 \%$ gel and then transferred to PVDF membranes (EMD Millipore). The membranes were blocked with $5 \%$ non-fat dried milk in TBS with $0.2 \%$ Tween-20 (TBST) buffer for $1 \mathrm{~h}$ at room temperature and then incubated with the corresponding primary antibodies overnight at $4^{\circ} \mathrm{C}$. The following primary antibodies were used: Cleaved-caspase-3 (cat. no. 9661; 1:1,000), cleaved-caspase-9 (cat. no. 9509; 1:1,000), cleaved-poly(ADP-ribose) polymerase (PARP; cat. no. 9548; 1:1,000), B-cell lymphoma-2 (Bcl-2; cat. no. 15071; 1:1,000), Bcl-2-associated X protein (Bax; cat. no. 5023; 1:1,000), phosphorylated extracellular signal-regulated kinase (p-ERK; cat. no. 4370; 1:2,000), ERK (cat. no. 4696; 1:2,000), p-p38 (cat. no. 4511; 1:1,000), p38 (cat. no. 8690; 1:1,000), c-Jun N-terminal kinase (JNK; cat. no. 9252; 1:1,000), p-JNK (cat. no. 4668; 1:1,000), cytochrome $c$ (cat. no. 11940; 1:1,000), Cytochrome $c$ oxidase IV (COX IV; cat. no. 4850; 1:1,000) and $\beta$-actin (cat. no. 3700; 1:1,000). After three washes with TBST, the membranes were subsequently incubated with anti-mouse IgG, HRP-conjugated antibody (cat. no. 7076; 1:5,000) or anti-rabbit IgG, HRP-conjugated antibody (cat. no. 7074; 1:5,000) for $1 \mathrm{~h}$ at room temperature. The protein signal was detected via electrochemiluminescence with an ECL-Plus kit (Beyotime Institute of Biotechnology). All antibodies were purchased from Cell Signaling Technology, Inc. All reagents were purchased from Amersham (Cytiva). Cell Mitochondria Isolation kit (Beyotime Institute of Biotechnology) was used to extract mitochondrial proteins in order to analyze cytochrome $c$.

Statistical analysis. The present results were obtained from $\geq 3$ independent experiments. Quantitative data are expressed as the mean \pm SD relative to the control value. Statistical analysis for multiple comparisons were performed using one-way ANOVA followed by Tukey's post hoc test. $\mathrm{P}<0.05$ was considered to indicate a statistically significant difference.

\section{Results}

Calycosin suppresses cell viability and promotes apoptosis of human osteosarcoma 143 B cells. Human osteosarcoma $143 \mathrm{~B}$ cells were exposed to calycosin at different concentrations for $48 \mathrm{~h}$ to check whether calycosin can inhibit the viability of osteosarcoma cells. As shown in Fig. 1A, following calycosin treatment for $48 \mathrm{~h}$, the activity of osteosarcoma 143B cells was inhibited in a dose-dependent manner $\left(\mathrm{IC}_{50}, 21.22 \pm 1.54 \mu \mathrm{g} / \mathrm{ml}\right)$. In addition, although the survival rate of hFOB1.19 cells decreased in a dose-dependent manner, the effect of calycosin on normal osteoblasts was negligible (Fig. 1A).

Induction of apoptosis is a key mechanism through which anticancer compounds exert their effects. Therefore, it was investigated whether the cytotoxicity of calycosin was associated with the induction of apoptosis in 143B cells. As shown in Fig. 1B, the flow cytometry assay results demonstrated that, after calycosin treatment for $48 \mathrm{~h}$, the percentage of apoptotic 
A

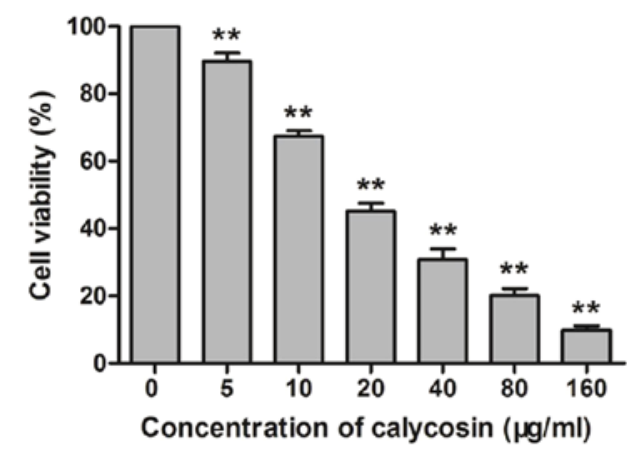

hFOB1.19

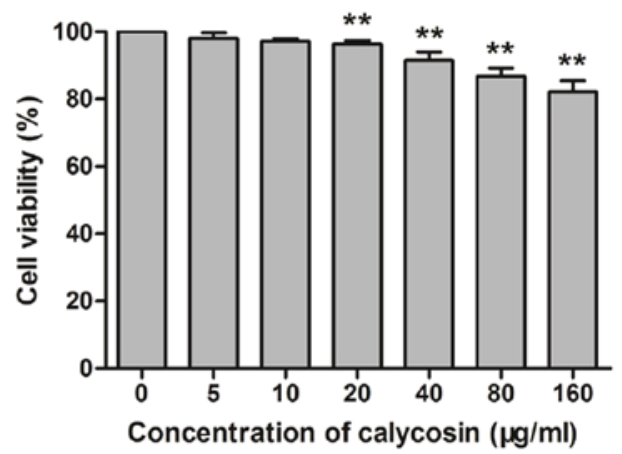

B
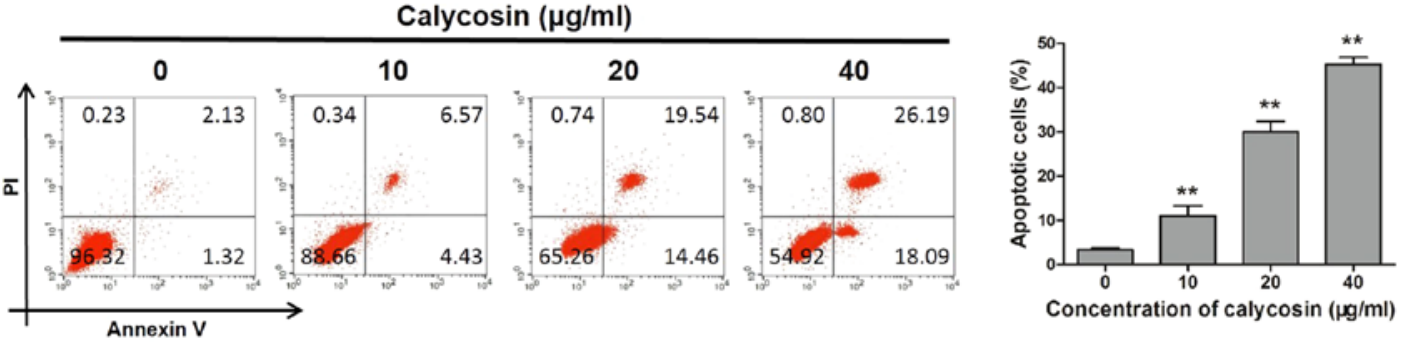

C
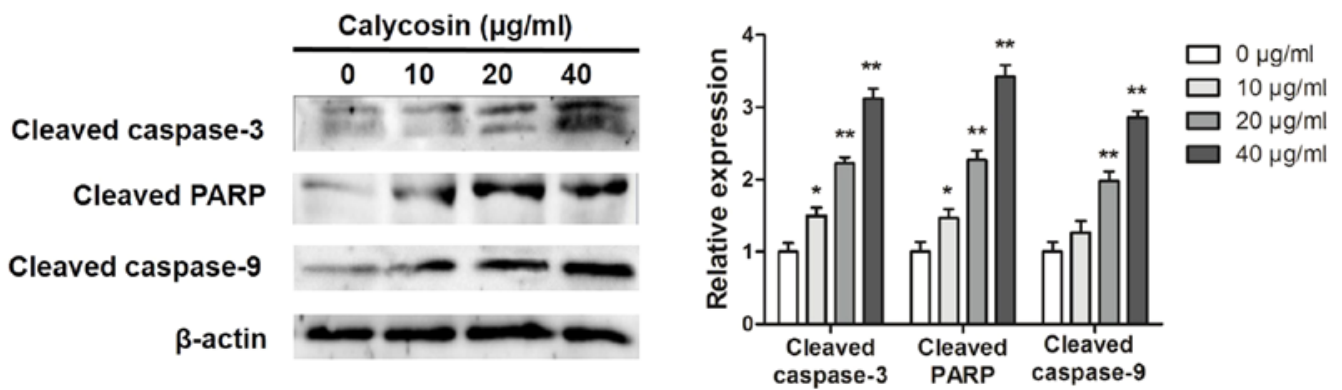

Figure 1. Calycosin suppresses cell viability and promotes apoptosis of human osteosarcoma 143B cells. 143B cells were treated with various concentrations of calycosin for 48 h. (A) Cell viability were examined by MTT assay. (B) Apoptosis was assessed by a flow cytometry assay (figures indicate the percentage of apoptotic cells in each quadrant in the flow cytometry plot). (C) Protein expression levels of cleaved caspase-3 and cleaved PARP were assessed by western blotting. ${ }^{*} \mathrm{P}<0.05,{ }^{* *} \mathrm{P}<0.01$ vs. $0 \mu \mathrm{g} / \mathrm{ml}$ calycosin. PARP, cleaved-poly(ADP-ribose) polymerase.

cells increased significantly in a concentration-dependent manner. As shown in Fig. 1C, western blotting revealed that calycosin significantly increased the protein expression levels of cleaved caspase-3, cleaved caspase-9, cleaved PARP, suggesting that calycosin induced apoptosis in 143B cells.

Calycosin regulates MMP in human osteosarcoma $143 \mathrm{~B}$ cells. The mitochondrial pathways are involved in the initiation and development of the apoptotic process (16). Therefore, the alterations in MMP and the expression of related proteins were investigated. Following exposure to calycosin, loss of MMP increased significantly in a dose-dependent manner in 143B cells, indicating that calycosin significantly increased the proportion of depolarized cells (Fig. 2A). In addition, calycosin increased the expression of Bax and the $\mathrm{Bax} / \mathrm{Bcl}-2$ ratio, whereas it decreased the expression of Bcl-2 (Fig. 2B). Furthermore, the levels of cytochrome $c$ were markedly reduced in the mitochondria and increased in the cytoplasm following calycosin treatment, verifying that calycosin-induced cell apoptosis was accompanied by mitochondrial dysfunction (Fig. 2C).
Calycosin activates p38-MAPK in human osteosarcoma $143 B$ cells. The MAPK signaling pathway regulates the apoptosis pathways (17). Western blotting was used to determine whether the MAPK signaling pathway was involved in calycosin-induced apoptosis of osteosarcoma cells. It was found that even at high concentrations, calycosin $(40 \mathrm{~g} / \mathrm{ml})$ had no effects on the phosphorylation of ERK1/2 and JNK (Fig. 3). By contrast, calycosin treatment significantly increased p-p38-MAPK in a dose-dependent manner (Fig. 3).

Calycosin inhibits the growth of human osteosarcoma $143 B$ cells via the p38-MAPK-mediated apoptotic pathway. To investigate the role of the p38 signaling pathway in calycosin-induced apoptosis, 143B cells were pretreated with the p38 inhibitor BIRB 796. The results of flow cytometry analysis demonstrated that BIRB 796 significantly ameliorated the calycosin-induced apoptosis of osteosarcoma cells (Fig. 4A). The results of the western blot analysis demonstrated that BIRB 796 treatment reversed the upregulated expression levels of p-p38 and proapoptotic proteins, including cleaved caspase- 3 and cleaved PARP, and the downregulated level of 
A
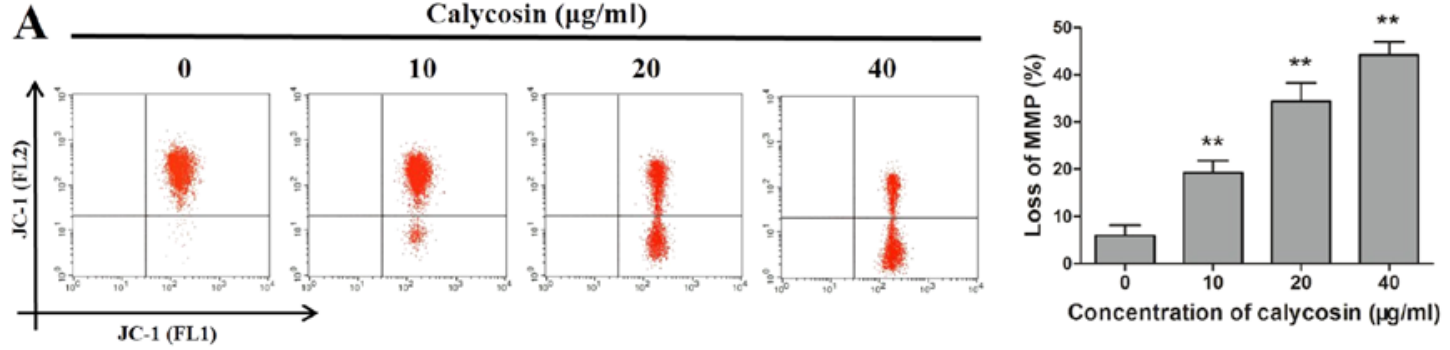

B

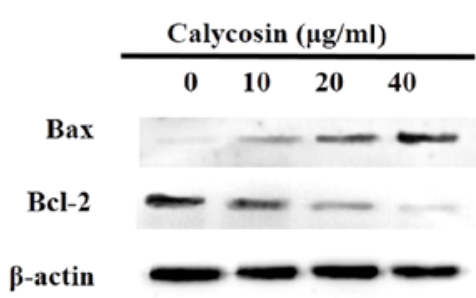

C

Cytoplasm

cytochrome $c$

$\beta$-actin

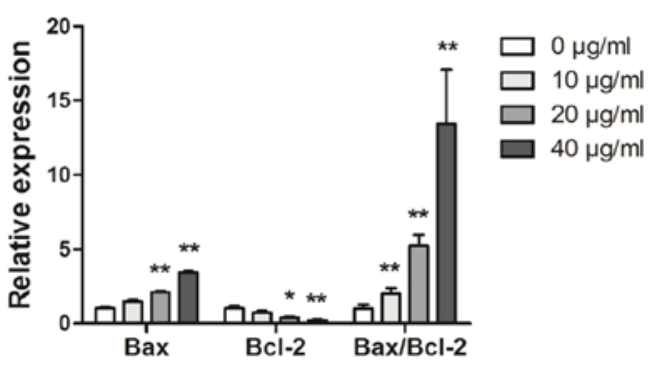

Mitochondria

cytochrome $c$

COX IV

Figure 2. Calycosin regulates MMP in human osteosarcoma 143B cells. 143B cells were treated with various concentrations of calycosin for $48 \mathrm{~h}$. (A) MMP was monitored using a JC-1 probe by a flow cytometry analysis. (B) Expression of mitochondrial-associated proteins was assessed using western blotting. (C) Calycosin-induced cytochrome $c$ release from mitochondria to cytoplasm was determined by immunoblotting analysis in $143 \mathrm{~B}$ cells. ${ }^{*} \mathrm{P}<0.05$, ${ }^{* *} \mathrm{P}<0.01$ vs. $0 \mu \mathrm{g} / \mathrm{ml}$ calycosin. MMP, mitochondrial membrane potential; Bax, Bcl-2-associated X protein; Bcl-2, B-cell lymphoma-2; COX IV, cytochrome $\mathrm{x}$ oxidase subunit 4 mitochondrial.
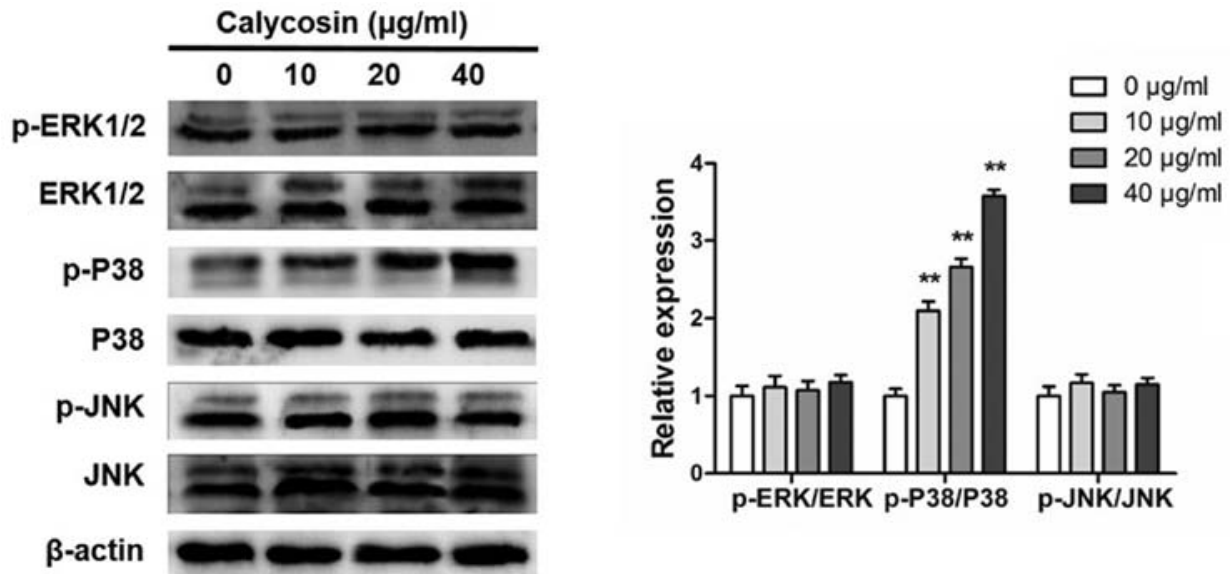

Figure 3. Calycosin activates p38-MAPK in human osteosarcoma 143B cells. 143B cells were treated with various concentrations of calycosin for $48 \mathrm{~h}$. The activation of the MAPK signaling pathway was assessed using western blotting. ${ }^{* *} \mathrm{P}<0.01 \mathrm{vs} .0 \mu \mathrm{g} / \mathrm{ml}$ calycosin. MAPK, mitogen-activated protein kinase; p-, phosphorylated; ERK, extracellular signal-regulated kinase; JNK, c-Jun N-terminal kinase.

the antiapoptotic protein Bcl-2, caused by calycosin treatment, whereas the total $\mathrm{p} 38$ protein levels remained constant during all treatments (Fig. 4B). The results of the flow cytometry analysis indicated that inhibition of p38 by BIRB 796 abolished the effects of calycosin treatment on the proportion of apoptotic and depolarized 143B cells (Fig. 4C and D). In summary, these results suggested that calycosin inhibited the growth of osteosarcoma cells via the intrinsic apoptosis pathway mediated by p38 signaling.

\section{Discussion}

Recent studies have extensively reported the anticancer and antimetastatic activities of calycosin in various types of cancer, 
A

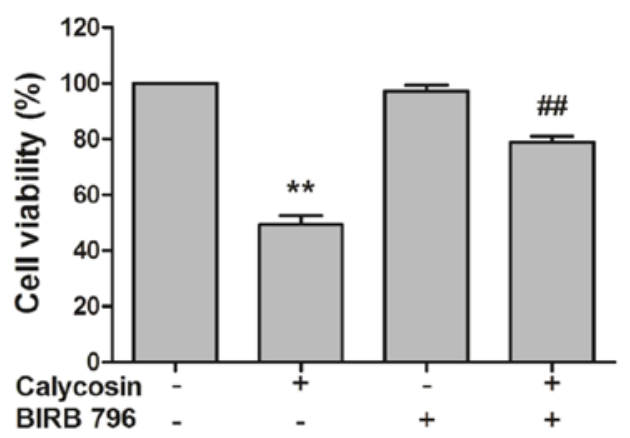

B

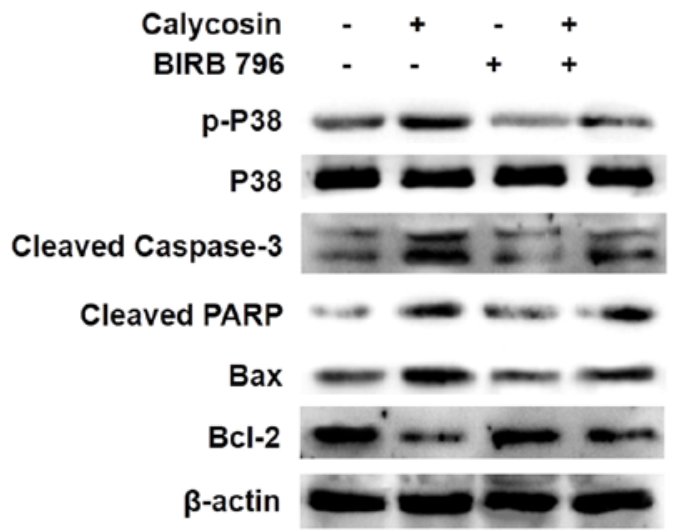

C
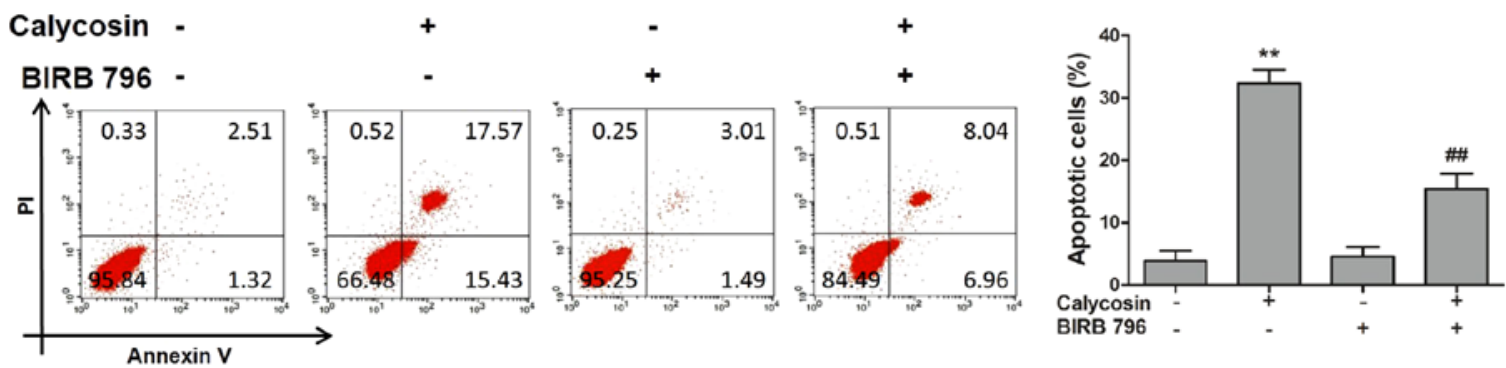

D
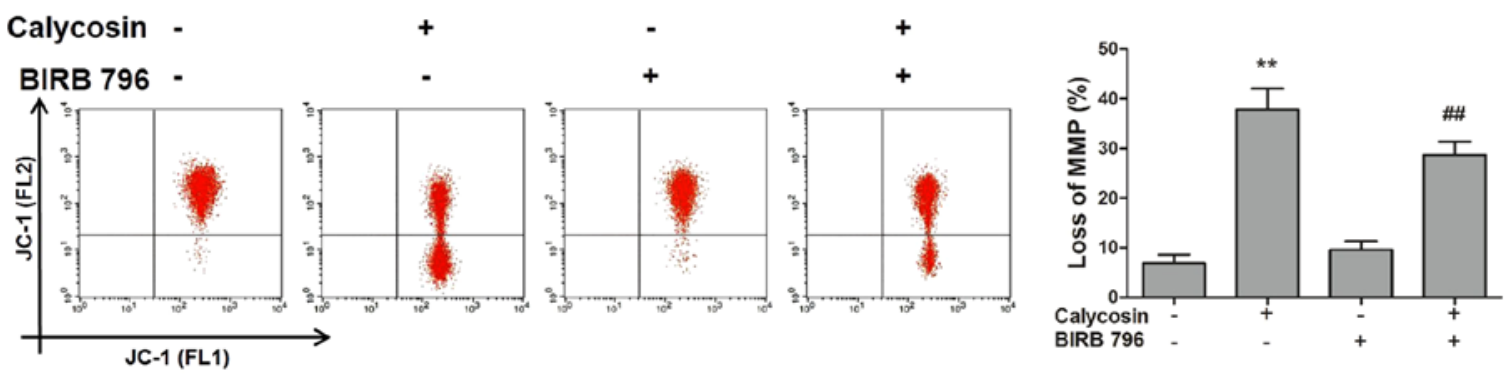

Figure 4. Calycosin inhibits the growth of human osteosarcoma 143B cells via the p38-MAPK-mediated apoptotic pathway. 143B cells were incubated with $1 \mu \mathrm{M}$ BIRB 796 for $2 \mathrm{~h}$, and then treated with calycosin $(20 \mu \mathrm{g} / \mathrm{ml})$ for $48 \mathrm{~h}$. (A) Cell viability was determined by an MTT assay. (B) Protein expression was analyzed using western blotting. (C) Apoptosis was assessed by a flow cytometry assay (figures indicate the percentage of apoptotic cells in each quadrant in the flow cytometry plot). (D) Expression of mitochondrial-associated proteins was assessed by flow cytometry analysis. ${ }^{* *} \mathrm{P}<0.01 \mathrm{vs} .0 \mu \mathrm{g} / \mathrm{ml}$ calycosin; ${ }^{\# \#} \mathrm{P}<0.01$ vs. $20 \mu \mathrm{g} / \mathrm{ml}$ calycosin. PARP, cleaved-poly(ADP-ribose) polymerase; MAPK, mitogen-activated protein kinase; p-, phosphorylated; Bax, Bcl-2-associated X protein; Bcl-2, B-cell lymphoma-2; MMP, mitochondrial membrane potential.

including breast cancer, colorectal cancer, ovarian cancer and nasopharyngeal carcinoma $(10,11,15)$. It was also reported that calycosin exerted an inhibitory effect on the growth of osteosarcoma (9). Sun et al (12) observed that calycosin induced apoptosis of estrogen receptor ${ }^{+}$MG-63 osteosarcoma cells via the PI3K/Akt/mTOR pathway. Qiu et al (18) demonstrated that calycosin was effective against osteosarcoma growth in vitro and in vivo via suppression of the miR-223-IкB $\alpha$ pathway in neoplastic cells. However, little is known about the mechanism underlying the role of calycosin in osteosarcoma. In the present study, it was examined whether calycosin inhibited osteosarcoma growth by activating the p38-MAPK pathway in osteosarcoma 143B cells.

Disruption of the apoptotic process plays an important role in the pathogenesis of tumors, and the cytotoxic effects of several antitumor drugs are often mediated by induction of apoptosis (19). Apoptosis is triggered by two major signaling pathways: The extrinsic pathway (also referred to as the death receptor pathway) and the intrinsic pathway (also referred to as the mitochondrial pathway) (20).

In the present study, calycosin at a concentration of $0-160 \mu \mathrm{g} / \mathrm{ml}$ was used for 24, 48 and $72 \mathrm{~h}$ to detect the inhibitory effect on osteosarcoma 143B cells. It was found that calycosin reduced the viability of osteosarcoma 143B cells in a concentration-dependent manner. Therefore, in the present study, a concentration of $20 \mu \mathrm{g} / \mathrm{ml}$, which was close to the $\mathrm{IC}_{50}$ concentration, were chosen to study the mechanism of action of calycosin on $143 \mathrm{~B}$ cells. However, smaller concentrations, such as 5 and $10 \mu \mathrm{g} / \mathrm{ml}$, also have good effects on 143B cells, so the specific mechanism of action using these concentrations still needs to be explored.

Previous reports have demonstrated that calycosin can inhibit cell growth and induce apoptosis of ovarian cancer cells by upregulating the expression of caspases and Bcl-2 family proteins $(11,12,15,18,21)$. 
Mitochondrial dependence signaling occurs through cleavage of caspase-9, which then activates downstream caspase-3, leading to cleavage of key cell substrates, including PARP, thereby inducing apoptosis (22). It was previously reported that calycosin exerts an anti-osteosarcoma effect, and its mechanism was associated with the activation of apoptosis (9). The present study also demonstrated that treatment with calycosin led to the cleavage of caspase-3, caspase- 9 and PARP in osteosarcoma 143B cells. Moreover, members of the Bcl-2 family play a key role in regulating mitochondrial membrane permeability (23).

The balance between proapoptotic Bax and antiapoptotic Bcl-2 is crucial for the determination of cell survival. The increased Bax/Bcl-2 ratio and the transfer of Bax from cytoplasm to the outer mitochondrial membrane renders cells more susceptible to apoptosis (24). It was previously reported that the release of cytochrome $c$ is primarily regulated by Bcl-2/Bax (25). The results of the present study demonstrated that calycosin led to an increase in the $\mathrm{Bax} / \mathrm{Bcl}-2$ ratio. Moreover, it was also observed that calycosin treatment resulted in a marked increase in the level of cytochrome $c$ in the cytoplasm and a dose-dependent decrease in the mitochondria, indicating that calycosin treatment caused cytochrome $c$ release from the mitochondria to the cytoplasm, further confirming the hypothesis that calycosin-induced apoptosis is mediated via the mitochondrial pathway.

In mammalian cells, the MAPK family is composed of ERK1/2, JNK, and p38 (26). In general, upregulation and activation of ERK1/2 and JNK promote tumor development, whereas p38 is a tumor suppressor. The role of p38-MAPK in cell proliferation and apoptosis has been extensively investigated (27). Accumulating evidence has suggested that the role of p38-MAPK is controversial in several tumor cells, and the variation may be associated with the applied stimuli and duration, as well as the specific characteristics and types of cells (28). The evidence revealed that NK007 induces G1/S cell cycle arrest by promoting p38-MAPK phosphorylation and suppressing the expression of hexokinase2, which is associated with acidification and oxygen consumption in ovarian cells (29). In vivo and in vitro studies demonstrated that serotonin yellow A induces apoptosis of HepG2 cells by significantly inhibiting the phosphorylation of the p38-MAPK pathway in hepatocellular carcinoma cells (30). Other findings have demonstrated that calycosin can inhibit growth and induce apoptosis in breast cancer cells via estrogen receptor $\beta$-dependent regulation of the insulin-like growth factor 1 receptor, p38 MAPK and PI3K/Akt pathways in breast cancer cells (15). In vitro and in vivo research demonstrated that licochalcone A regulated the mitochondrial-mediated internal apoptotic pathway via p38-MAPK, and exerted antitumor effects on human osteosarcoma cells (28). Similarly, the present study suggested that calycosin increased p-p38-MAPK expression, whereas a specific p38 inhibitor, BIRB 796, significantly reversed the effects of calycosin treatment on the viability, caspase expression, MMP and apoptotic rate of osteosarcoma 143B cells. These results indicated that calycosin-induced apoptosis may be caused by the activation of the p38-MAPK pathway-mediated mitochondrial apoptotic pathway in human osteosarcoma cells (Fig. 5). However, additional studies are required to examine the molecular mechanisms through

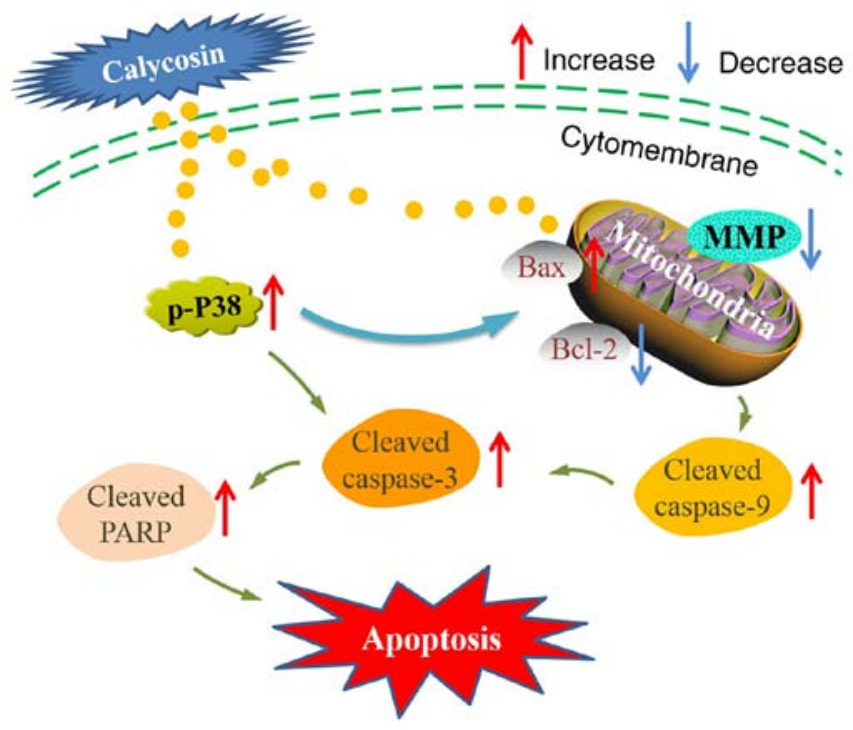

Figure 5. Schematic diagram showing the proposed mechanism of action of calycosin. Underlying mechanism of calycosin-induced apoptotic cell death via the activation of $\mathrm{p} 38$-mitogen-activated protein kinase pathway-mediated mitochondrial pathways of apoptosis in human osteosarcoma cells. PARP, cleaved-poly(ADP-ribose) polymerase; p-, phosphorylated; Bax, Bcl-2-associated X protein; Bcl-2, B-cell lymphoma-2; MMP, mitochondrial membrane potential.

which calycosin regulates the mitochondrial-related apoptotic pathways that control the interaction between $\mathrm{p} 38$-MAPK and apoptosis induction.

In conclusion, calycosin significantly induced mitochondrial apoptosis in human osteosarcoma cells in vitro, as indicated by the increased level of cleaved caspase-3 and -9 and PARP protein expression, and $\mathrm{Bax} / \mathrm{Bcl}-2$ ratio. Notably, activation of caspases mediated by $\mathrm{p} 38$-MAPK or increased expression of mitochondrial-related proteins may promote mitochondrial apoptosis in human osteosarcoma cells. These results provided insights into the potential value of calycosin in the clinical treatment of osteosarcoma.

\section{Acknowledgements}

Not applicable.

\section{Funding}

No funding was received.

\section{Availability of data and materials}

The datasets used and/or analyzed during the current study are available from the corresponding author on reasonable request.

\section{Authors' contributions}

WT performed the majority of the experiments and drafted the manuscript. ZWW helped perform the experiments. BMY analyzed the data and drafted the manuscript. YGB conceived and designed the study, supervised the experiments and edited the manuscript. All authors read and approved the final manuscript. 


\section{Ethics approval and consent to participate}

Not applicable.

\section{Patient consent for publication}

Not applicable.

\section{Competing interests}

The authors declare that they have no competing interests.

\section{References}

1. Mirabello L, Troisi RJ and Savage SA: Osteosarcoma incidence and survival rates from 1973 to 2004: Data from the surveillance, epidemiology, and end results program. Cancer 115: 1531-1543, 2009.

2. Eyre R, Feltbower RG, Mubwandarikwa E, Eden TO and McNally RJ: Epidemiology of bone tumours in children and young adults. Pediatr Blood Cancer 53: 941-952, 2009.

3. Harrison DJ, Geller DS, Gill JD, Lewis VO and Gorlick R Current and future therapeutic approaches for osteosarcoma. Expert Rev Anticancer Ther 18: 39-50, 2018.

4. Baudino TA: Targeted cancer therapy: The next generation of cancer treatment. Curr Drug Discov Technol 12: 3-20, 2015.

5. Tao Y, Wang Y, Wang X, Wang C, Bao K, Ji L, Jiang G and Hong M: Calycosin suppresses epithelial derived initiative key factors and maintains epithelial barrier in allergic inflammation via TLR4 mediated NF- $\kappa$ B pathway. Cell Physiol Biochem 44 1106-1119, 2017.

6. Dong L, Yin L, Chen R, Zhang Y, Hua S, Quan H and Fu X: Anti-inflammatory effect of Calycosin glycoside on lipopolysaccharide-induced inflammatory responses in RAW 264.7 cells. Gene 675: 94-101, 2018.

7. Duan X, Meng Q, Wang C, Liu Z, Liu Q, Sun H, Sun P, Yang X, Huo X, Peng J and Liu K: Calycosin attenuates triglyceride accumulation and hepatic fibrosis in murine model of non-alcoholic steatohepatitis via activating farnesoid $\mathrm{X}$ receptor. Phytomedicine 25: 83-92, 2017.

8. Liu Y, Che G, Di Z, Sun W, Tian J and Ren M: Calycosin-7-O- $\beta$-D-glucoside attenuates myocardial ischemia-reperfusion injury by activating JAK2/STAT3 signaling pathway via the regulation of IL-10 secretion in mice. Mol Cell Biochem 463: 175-187, 2020.

9. Qiu R, Li X, Qin K, Chen X, Wang R, Dai Y, Deng L and Ye Y: Antimetastatic effects of calycosin on osteosarcoma and the underlying mechanism. Biofactors 45: 975-982, 2019.

10. Wu G, Niu M, Qin J, Wang Y and Tian J: Inactivation of Rab27B-dependent signaling pathway by calycosin inhibits migration and invasion of ER-negative breast cancer cells Gene 709: 48-55, 2019.

11. El-Kott AF, Al-Kahtani MA and Shati AA: Calycosin induces apoptosis in adenocarcinoma HT29 cells by inducing cytotoxic autophagy mediated by SIRT1/AMPK-induced inhibition of Akt/mTOR. Clin Exp Pharmacol Physiol 46: 944-954, 2019.

12. Sun H, Yin M, Qian W and Yin H: Calycosin, a phytoestrogen isoflavone, induces apoptosis of estrogen receptor-positive MG-63 osteosarcoma cells via the phosphatidylinositol 3-kinase (PI3K)/AKT/mammalian target of rapamycin (mTOR) pathway. Med Sci Monit 24: 6178-6186, 2018.

13. Zhang W and Liu HT: MAPK signal pathways in the regulation of cell proliferation in mammalian cells. Cell Res 12: 9-18, 2002.

14. Ning L, Wan S, Jie Z, Xie Z, Li X, Pan X, Wan X, Chen W, Huang H, Wang J, et al: Lycorine induces apoptosis and G1 phase arrest through ROS/p38 MAPK signaling pathway in human osteosarcoma cells in vitro and in vivo. Spine (Phila Pa 1976) 45 E126-E139, 2019.
15. Chen J, Hou R, Zhang X, Ye Y, Wang Y and Tian J: Calycosin suppresses breast cancer cell growth via ER $\beta$-dependent regulation of IGF-1R, p38 MAPK and PI3K/Akt pathways. PLoS One 9: e91245, 2014

16. Zhang B, Wang D, Guo F and Xuan C: Mitochondrial membrane potential and reactive oxygen species in cancer stem cells. Fam Cancer 14: 19-23, 2015.

17. Wada T and Penninger JM: Mitogen-activated protein kinases in apoptosis regulation. Oncogene 23: 2838-2849, 2004.

18. Qiu R, Ma G, Li X, Shi Q, Li X, Zhou X, Tang Y, Xie Z, Liao S, Qin Y, et al: Clinical case report of patients with osteosarcoma and anticancer benefit of calycosin against human osteosarcoma cells. J Cell Biochem 120: 10697-10706, 2019.

19. Fleischer A, Ghadiri A, Dessauge F, Duhamel M, Rebollo MP, Alvarez-Franco F and Rebollo A: Modulating apoptosis as a target for effective therapy. Mol Immunol 43: 1065-1079, 2006.

20. Creagh EM: Caspase crosstalk: Integration of apoptotic and innate immune signalling pathways. Trends Immunol 35: 631-640, 2014

21. Zhou Y, Liu QH, Liu CL and Lin L: Calycosin induces apoptosis in human ovarian cancer SKOV3 cells by activating caspases and Bcl-2 family proteins. Tumour Biol 36: 5333-1339, 2015.

22. Palmer CS, Osellame LD, Stojanovski D and Ryan MT: The regulation of mitochondrial morphology: Intricate mechanisms and dynamic machinery. Cell Signal 23: 1534-1545, 2011.

23. Xiong S, Mu T, Wang G and Jiang X: Mitochondria-mediated apoptosis in mammals. Protein Cell 5: 737-749, 2014.

24. Siu WP, Pun PB, Latchoumycandane C and Boelsterli UA: Bax-mediated mitochondrial outer membrane permeabilization (MOMP), distinct from the mitochondrial permeability transition, is a key mechanism in diclofenac-induced hepatocyte injury: Multiple protective roles of cyclosporin A. Toxicol Appl Pharmacol 227: 451-461, 2008.

25. Jergens A, Young J, Moore D, Wang C, Hostetter J, Augustine L, Allenspach K, Schmitz S and Mosher C: Bcl-2/Caspase 3 mucosal imbalance favors $\mathrm{T}$ cell resistance to apoptosis in dogs with inflammatory bowel disease. Vet Immunol Immunopathol 158: $167-74,2014$

26. Xu T, Wang NS, Fu LL, Ye CY, Yu SQ and Mei CL: Celecoxib inhibits growth of human autosomal dominant polycystic kidney cyst-lining epithelial cells through the VEGF/Raf/MAPK/ERK signaling pathway. Mol Biol Rep 39: 7743-7753, 2012.

27. Cuenda A and Rousseau S: p38 MAP-kinases pathway regulation, function and role in human diseases. Biochim Biophys Acta 1773: 1358-1375, 2007.

28. Lin RC, Yang SF, Chiou HL, Hsieh SC, Wen SH, Lu KH and Hsieh YH: Licochalcone a-induced apoptosis through the activation of p38MAPK pathway mediated mitochondrial pathways of apoptosis in human osteosarcoma cells in vitro and in vivo. Cells 8: 1441, 2019.

29. Li Z, Tang X, Luo Y, Chen B, Zhou C, Wu X, Tang Z, Qi X, Cao G, Hao J, et al: NK007 helps in mitigating paclitaxel resistance through p38MAPK activation and HK2 degradation in ovarian cancer. J Cell Physiol: Feb 20, 2019 (Epub ahead of print). doi: $10.1002 /$ jcp.28278.

30. Zhang J, Li J, Song H, Xiong Y, Liu D and Bai X: Hydroxysafflor yellow A suppresses angiogenesis of hepatocellular carcinoma through inhibition of p38 MAPK phosphorylation. Biomed Pharmacother 109: 806-814, 2019.

This work is licensed under a Creative Commons Attribution-NonCommercial-NoDerivatives 4.0 International (CC BY-NC-ND 4.0) License. 